\title{
Impacto de las TIC en las representaciones sociales de los docentes en la modalidad b-learning ${ }^{1}$ \\ Impact of ICT in teachers' social representations in the b-learning mode
}

Recibido: 18 de marzo de 2016 - Revisado: 24 de agosto de 2016 - Aceptado: 19 de octubre de 2016.

José Eduardo Padilla Beltrán ${ }^{2}$

Wilmer Hernando Silva Carreño ${ }^{3}$

\section{Resumen}

Este artículo resultado de investigación da cuenta del sentido, alcance e impacto que tienen las tecnologías de la información y la comunicación (TIC) en la configuración de aquellas representaciones sociales de los docentes que gestionan y orientan procesos de formación en la modalidad b-learning en educación superior. Mediante entrevistas semiestructuradas, la configuración de los resultados se desarrolló por medio de la triangulación hermenéutica como proceso metodológico, y desde esta se analizó el impacto de las TIC en dichas representaciones sociales desde un enfoque de la pedagogía sociocrítica. Se concluye que la modalidad b-learning requiere una fundamentación conceptual y epistemológica propia, más allá de su operatividad, es decir, lejos está de comprenderse como una simple mediación tecnológica del conocimiento y del aprendizaje.

\section{Palabras clave}

B-learning, TIC, docente, representaciones sociales, pedagogía sociocrítica.

\begin{abstract}
This research article gives an account of the meaning, scope and impact of information and communication technologies (ICT) in the configuration of those social representations of teachers who manage and guide training processes in the b-learning mode in higher education. Through semi-structured interviews, the configuration of the results was developed through hermeneutical triangulation as a methodological process, and from this the impact of ICT in these social representations was analyzed from a socio-critical pedagogy approach. It is concluded that the b-learning modality requires its own conceptual and epistemological foundation, beyond its operability, that is, it is far from
\end{abstract}

Keywords

B-learning, ICT, teacher, social representations, socio-critical pedagogy.

\footnotetext{
1 Artículo resultado del proyecto de investigación "Representaciones sociales sobre el rol del docente tutor en la Facultad de Estudios a Distancia de la Universidad Militar Nueva Granada" (DIS 1754). Financiado por la Vicerrectoría de Investigaciones en la vigencia 2015 .

${ }^{2}$ Docente asociado, director del Centro de Investigaciones de la Facultad de Estudios a Distancia y director del Grupo PYDES de la Universidad Militar Nueva Granada. Licenciado en Matemáticas, Universidad Pedagógica Nacional, Colombia. Especialista en Orientación Educativa, Universidad Manuela Beltrán. Magíster en Administración y Supervisión Educativa, Universidad Externado de Colombia. Magíster en Educación con énfasis en Evaluación Educativa, Universidad Santo Tomás, Bogotá, Colombia. Doctor en Educación, Newport University, EE UU. Postdoctor en Ciencia Sociales, Educación e Interculturalidad, Universidad Santo Tomás.

Correo electrónico: eduardo.padilla@ unimilitar.edu.co

${ }^{3}$ Docente de la Universidad de San Buenaventura, Bogotá, Colombia y de la Secretaría de Educación Distrital. Licenciado en Filosofía y Magíster en Ciencias de la Educación, Universidad de San Buenaventura, Bogotá, Colombia. Doctor en Educación, Universidad Pedagógica Nacional, Bogotá, Colombia. Investigador del Grupo PYDES.

Correo electrónico: wsilva@usbbog. edu.co

Para citar este artículo use: Padilla, J., \& Silva, W. (2017). Impacto de las TIC en las representaciones sociales de los docentes en la modalidad blearning. Civilizar Ciencias Sociales y Humanas, 17(32), 161-170. Doi: $10.22518 / 16578953.824$
} 


\section{Introducción}

La investigación en torno a las representaciones sociales de los docentes en la modalidad blended learning, tuvo como asunto particular de análisis el hecho de que a éstas convergen categorías nucleares como la formación pedagógica e investigativa del docente y, especialmente, el acceso, uso y la apropiación didáctica de las tecnologías de la información y la comunicación (TIC).

Con relación a esta última categoría convergente, se hace necesario indagar por la cohesión entre las prácticas educativas mediadas por las TIC y los procesos de alfabetización y educación virtual de estas estrategias. Asimismo, constituye asunto de comprensión entrever el lugar o no, y el sentido que esto trae, de un posible o eventual desplazamiento del rol docente como enseñante para que otros profesionales adquieran ese rol desde perspectivas técnicas, de gestión y administración de la pedagogía, la didáctica y otros componentes y conceptos propios de la práctica docente.

El siglo XXI ha traído consigo cambios para el mundo universitario, en los cuales las transformaciones económicas y políticas constituyen una información importante para reflexionar sobre su estado actual. Como afirma Villanueva (2010): "La educación superior deberá ser objeto pero también sujeto de esas políticas de desarrollo" (p. 97), y aunque puede indicarse que la globalización no se desarrolla en función de las necesidades y condiciones locales o regionales de la sociedad, es importante que cada país con vistas a su sociedad y a las demandas de esta, busque el avance de nuevas alternativas, mejorando el sistema, maximizando los recursos con los que se cuenta y apuntando al desarrollo sostenible para todos sus ciudadanos.

De esta manera, en los últimos años la educación superior ha procurado comprender estos cambios y cada vez son más las ofertas académicas de educación a distancia y/o virtual en la educación superior, permitiendo abrir nuevos escenarios al servicio de los estudiantes a través de proyectos tecnológicos y herramientas que permiten organizar nuevas formas de encuentro entre el maestro y el estudiante. Brunner (1999) señala:

La educación, en particular, en todo este nuevo contexto informacional representa mucho más que la posibilidad de usar con fines pedagógicos las nuevas tecnologías [...] De hecho, no es el hardware ni el software lo que está causando la revolución en curso, sino el empleo social de las tecnologías, su uso para reconfigurar la sociedad (p. 87).

\section{Método: las unidades discursivas como proceso}

La entrevista semiestructurada que se manejó tuvo como objetivo recoger información acerca de la dimensión representacional de los docentes sobre su rol en modalidades de aprendizaje $b$-learning y virtual, tomando como referente principal la Facultad de Estudios a Distancia de la Universidad Militar Nueva Granada y la participación de docentes de otras instituciones universitarias del ámbito nacional e internacional. El proceso de la recolección de la información, que tuvo como instrumento el antes enunciado, se desarrolló desde un enfoque cualitativo a través del método de comparación constante.

La población informante se seleccionó de forma aleatoria entre docentes de educación superior en los niveles de pregrado y posgrado de distintas universidades nacionales e internacionales, que desarrollan procesos de educación en la modalidad b-learning. Esto dado que el proyecto de investigación tenía como objetivo principal analizar referentes vivenciales para fundar perspectivas teóricas (Silva, 2016 , p. 32) ${ }^{1}$ que permitan una comprensión coherente y una prospectiva oportuna de esta modalidad. 
La información suministrada por los participantes fue sometida a un proceso de arbitraje mediante su sistematización, categorización y codificación conceptual, en matrices conceptuales, a partir de los tres núcleos conceptuales del proyecto de investigación. Para el proceso de análisis de la información se recurre a la hermenéutica como método, especialmente, en el tránsito de la explicación a la comprensión (Ricoeur, 2008), como un modo concreto para interpretar y construir teoría o nuevo conocimiento.

Dicho tránsito se desarrolló mediante la triangulación hermenéutica (Oppermann, 2000), en el sentido en que convergen diversos elementos en el ejercicio de la interpretación para la construcción de teoría, por lo que se comprende esta forma de triangular como un cruce dialéctico de la información con el referente teórico (Cisterna, 2005, p. 68).

En este sentido, la interpretación de la información se realizó por medio del proceso de triangulación hermenéutica, en la que, luego de reunir la información obtenida en la investigación, se cruza la información proporcionada por los informantes, estableciendo relaciones de comparación por categorías, lo que determina las conclusiones categoriales para, finalmente, cruzar las conclusiones con el marco teórico; y de este modo, una vez realizada la triangulación, obtenemos lo que se denomina como resultados de la investigación (Cisterna, 2005, pp. 69-70). Para el proyecto se asume como base teórica o referente conceptual lo enunciado por Moscovici (1979) en torno a las representaciones sociales y la perspectiva de la pedagogía sociocrítica desde H. Giroux y P. McLaren.

Como resultado de este proceso, se consolidan lo que puede denominarse una unidad hermenéutica de comprensión o la configuración de unidades discursivas como unidades de comprensión o de interacción discursiva (Silva, 2016, pp. 40-41). Como unidad discursiva se identificó que las tecnologías de la información y la comunicación devienen como un objeto o elemento sustantivo de las representaciones sociales de los docentes en los contextos b-learning.

\section{Marco referencial}

\section{La teoría de la representación social.}

Especialmente en la educación b-learning confluyen elementos particulares de constitución, por ejemplo, el análisis y la forma como se constituye el espacio virtual y común de trabajo, los repertorios lingüísticos o universos semánticos producidos por los participantes que contienen elementos significativos y que involucran una dimensión cognitiva, simbólica y emocional.

La teoría de la representación social es un generador de un amplio campo de debates, intercambios y disenso dentro de la pregunta por lo social. Hay un aumento considerable en el número de investigadores dedicados al tema, así como las diferentes conexiones que se despliegan y se establecen con varios campos del saber.

De alguna forma esto tiene que ver con una perspectiva psicológica de lo social, y para este estudio toma como referente la noción de representación social que caracteriza de base una posición conjunta transversada por diversidad de conceptos sociológicos $\mathrm{y}$ psicológicos que funda, de algún modo, una forma particular de emergencia social (Moscovici, 1979, p. 41). Puede decirse, las representaciones sociales son una construcción colectiva, multifacética y polimorfa, que tienen como base diversos elementos simbólicos que son generados por los sujetos sociales en situaciones de interacción con el contexto en que viven y que construyen.

\section{La pedagogía sociocrítica y las TIC.}

Como se indicó páginas atrás, se considera que la configuración de las representaciones 
sociales de los docentes puede sedimentarse a partir de una comprensión sociocrítica de la educación y la pedagogía. A esto converge de forma concreta el aporte del teórico Henry Giroux (1990), quien cuestiona la formación misma de los docentes, a quienes se les entrega metodologías que parecen negar el pensamiento crítico, perdiendo el interés de examinar la naturaleza subyacente de los problemas escolares (p. 78). Esta apreciación indica tener en cuenta la metodología utilizada, que permita e incite un pensamiento crítico al otro lado de la pantalla, respecto a la información que se encuentra y los aportes que tiene para su aprendizaje.

Para Giroux (1990) las escuelas introducen y legitiman formas particulares de vida social, encarnan y expresan una cierta lucha sobre qué formas de autoridad, tipos de conocimiento, regulación moral e interpretaciones del pasado y del futuro deberían ser legitimadas y transmitidas a los estudiantes; frente al amplio despliegue de diversos fenómenos que necesariamente configuran la escuela del futuro, la escuela digital (p. 98).

Giroux (2001) plantea que la universidad está realizando acciones tendientes a estar actualizada en la implementación y uso de los recursos virtuales y digitales en esta nueva revolución digital y que formar a los estudiantes en el uso de estas herramientas es primordial, como también lo es que ellos sepan leer y analizarlos de manera crítica (p. 46). Se pregunta: “¿cómo pueden los profesores replantear la educación, en vista de las nuevas formas de pedagogía cultural que han surgido fuera de la enseñanza tradicional?" (pp. 38-39). Entonces, los docentes están llamados a actuar no solamente en su área de conocimiento, sino, también, frente a las subjetividades y culturas actuales, es decir, que conjugando la educación con la introducción de tecnologías pueda tener una mirada reflexiva y crítica de la situación sociocultural del contexto (Silva, 2011, p. 49).
Así, desde la pedagogía crítica la escuela no es un punto neutral y por ende los docentes tampoco pueden adoptar una postura neutral. La escuela "debe comprenderse como un espacio donde se ha de formar para comprender la realidad" (Silva, 2011, p. 50), por lo que emerge la importancia de que tanto los docentes como los profesores deben ser críticos, activos y trasformativos en cada uno de los contextos de sus prácticas.

\section{Resultados y discusión}

En esta sección se presenta, a modo de unidades de comprensión y construcción de sentido, los elementos y las discusiones que surgieron a partir del análisis de las interacciones discursivas y de las intervenciones de los participantes.

El contexto de socialización de la investigación y de debate en torno a la educación b-learning, que se tomó como núcleo para este trabajo y la participación de profesores e investigadores en torno a este campo particular, resultó oportuno para identificar las interacciones discursivas que se configuraron según lo informado por los participantes en relación con el lugar y el sentido que tienen las tecnologías y su apropiación.

A continuación se desarrollan cuatro aristas de reflexión derivadas del proceso investigativo. En cada una se presenta en una tabla, solo a modo de ejemplo, la puesta en confrontación de la información obtenida a partir de alguno de los participantes con la comprensión emergente desde la perspectiva sociocrítica.

\section{Las TIC como dispositivo del pen- samiento crítico en los contextos $b$ - learning.}

La educación virtual ha estado presente por medio del avance tecnológico de la comunicación, especialmente de las redes sociales. 
Las opiniones de diferentes educadores y profesionales en investigación, de instituciones internacionales, así como expertos en el ejercicio mismo de la educación $b$ - learning, permiten realizar reflexiones respecto al rol del docente en el uso de las TIC, la educación b-learning y el pensamiento crítico del estudiante en esta modalidad de aprendizaje.

Aceptar e incluir esta modalidad de aprendizaje ha sido cuestionada, dados los "hábitos" culturales y pedagógicos que hay en los procesos de la educación. Los participantes permiten destacar que la inclusión de las TIC en los procesos de enseñanza por parte de los docentes ha generado una forma de reacción "alérgica" que se da naturalmente a lo desconocido y que implica las diferentes aplicaciones tecnológicas. Reconocen cómo por medio de la pantalla y los programas de televisión formativos, las TIC estuvieron presentes desde hace muchos años atrás, siendo así el primer dispositivo de conexión por el cual la experiencia con las TIC ha posibilitado no solo enseñar, sino también aprender, convirtiendo la educación en un co-aprendizaje.

Esto es precisamente lo que da cuenta de las posibilidades de interactuar e intercambiar diferentes percepciones de aprendizaje y conocimiento al que hay acceso y del ejercicio transdisciplinar en el uso de las TIC.

Barbas (2014) sugiere tener en cuenta la necesidad de establecer planteamientos en educación y comunicación que den respuesta a los nuevos retos respecto a las dificultades de transformar los modelos de formación de los docentes (p. 23). Y es precisamente el ajuste al cambio social y cultural que el medio demanda o reclama del docente estrategias innovadores y pertinentes en el campo educativo. Esto indica la necesidad de innovación didáctica y pedagógica de los docentes, así como el empoderamiento con relación al manejo de las TIC.

Tabla 1.

B-learning y pensamiento crítico

Informante-Actor.

"[...] no puedes desarrollar habilidades de pensamiento crítico [en las] personas que no quieran responder preguntas. Sin duda, primero tienes que motivarlos de una forma $\mathrm{u}$ otra a querer responder preguntas. Luego, la forma en que se hace es explorando formas diferentes de pensar y resolver el problema. Eso envuelve pensamiento crítico" (Participante 4, comunicación personal, 13 de junio de 2016).

Fuente: elaboración propia.

Conforme lo indican los participantes, el pensamiento crítico del estudiante y del docente frente al uso de las TIC en su proceso de aprendizaje, no implica necesariamente alejarse de la forma tradicional de enseñanza. Los participantes destacan, por un lado, la importancia de que la práctica educativa de los docentes les permita a los estudiantes resolver problemas y responder preguntas, donde ellos tengan diferentes posibilidades para responder, sin ser el docente quien exija una determinada y única respuesta y forma de hacerlo. Por otro lado, en toda práctica un mal uso de las TIC puede generar la configuración de hábitos inapropiados, como es el caso del copy and paste en Internet por parte de los estudiantes. Esto lejos está de promover el pensamiento crítico y reflexivo, o lo que es, la construcción del saber.

Para los participantes el pensamiento crítico permite a los estudiantes resolver problemas y este ejercicio se convierte en su propio conocimiento. Resaltan la importancia de diseñar y ejecutar estrategias oportunas por parte del docente para desarrollar el pensamiento crítico en los estudiantes. Asimismo, para que los estudiantes desarrollen habilidades para pensar y responder a aquellas situaciones y contextos que reclaman un empoderamiento, especialmente, de orden político. 


\section{Impacto semántico de las TIC en la configuración representacional del docente.}

La configuración del rol de los docentes está considerablemente permeada por la comprensión o perspectiva que el estudiante va elaborando en su experiencia de formación. En este sentido, para los participantes, en general, los estudiantes aprecian o valoran más al docente que media sus clases con herramientas tecnológicas, y propiamente, en el caso de los contextos b-learning, que dinamiza dicha mediación.

Sin embargo, para algunos participantes, puede correrse el riesgo de caer en una buena alfabetización en el uso y apropiación de los recursos tecnológicos por parte de los profesores, al margen de una sólida disciplinariedad o experticia en los asuntos problemáticos y temáticos del área de conocimiento. En este aspecto, resulta explícitas afirmaciones como: "El profesor que desarrolla sus clases de manera virtual no es un experto en su área de conocimiento, posee menos dominio de los temas". Esto hace que se vaya alimentando peyorativamente la consideración de que el docente en contextos presenciales tiene un mayor estatus en cuanto a los conocimientos de la disciplina, aun cuando se vean impelidos por los estudiantes al uso de herramientas tecnológicas.

Por otra parte, es necesario trasponer aquellos vicios de comprensión que se han venido configurando alrededor del valor formal y legal de los procesos de formación en contextos b-learning, y en general, en educación a distancia y/o virtual. Tal es el caso de una posible subvaloración hacia esta modalidad al considerar en el medio cultural que los títulos académicos obtenidos y la misma formación tienen menos validez que los presenciales. Esto constituye un reto semántico al que deben dar desarrollo, en primer lugar, tanto los profesores como las instituciones, y por extensión la sociedad.
Tabla 2.

Representación del docente

\begin{tabular}{|c|c|}
\hline Informante-Actor. & Perspectiva crítica \\
\hline $\begin{array}{l}\text { "El profesor que solo se de- } \\
\text { dica a la docencia y no inves- } \\
\text { tiga es menos valorado en el } \\
\text { ámbito académico" (Partici- } \\
\text { pante } 6 \text {, comunicación perso- } \\
\text { nal, } 24 \text { de mayo de } 2016 \text {. }\end{array}$ & $\begin{array}{l}\text { "La pedagogía crítica necesita } \\
\text { crear nuevas formas de } \\
\text { conocimiento mediante su } \\
\text { énfasis en la desintegración } \\
\text { de los límites disciplinarios y } \\
\text { la creación de nuevos espacios } \\
\text { donde ese conocimiento pueda } \\
\text { producirse" (Giroux, 2003, p. } \\
\text { 308). }\end{array}$ \\
\hline
\end{tabular}

Fuente: elaboración propia.

La información brindada por el actor 6 denota la necesidad de fortalecer y promover tanto la formación en investigación como el desarrollo de proyectos de investigación, especialmente, relacionados con la caracterización propia de los contextos b-learning. Desde la pedagogía crítica se desprende de hecho tal necesidad de crear nuevos espacios para la producción del saber.

De parte de las instituciones, les compete revisar el estatus y el sentido que tiene toda estrategia de educación a distancia y/o virtual, de modo que, por ejemplo, financieramente se les apoye. Justamente, una de las afirmaciones de los participantes resulta de interés, a saber: "en el nuevo estatuto docente los estímulos económicos que se dan por investigar o formarse en el uso de las tecnologías son mínimos".

Por otra parte, llama la atención el impacto semántico que tienen las políticas reglamentarias en el contexto nacional, especialmente, en torno a la actividad investigativa. Es un reto en este aspecto no dar cabida a una comprensión equívoca del ejercicio de investigación como aquella que determina la calidad de la docencia. Como indican varios participantes, existe la tendencia a considerar que un maestro investigador solo tiene como elemento diferenciador y motivador la búsqueda de "puntos" por el acto de investigar y debe dedicarse a la docencia por la necesidad de un salario. 


\section{La "alfabetización" tecnológica en la práctica pedagógica.}

Tabla 3.

Alfabetización y práctica docente

\section{Informante-Actor.}

"El profesor debe comprender que las tecnologías son solo un 'medio' que no resolverá todas sus dificultades y que es su labor hacer buen uso de ellas planteando estrategias didácticas diversas" (Participante 12, comunicación personal, 13 de junio de 2016).

Fuente: elaboración propia.

Analizar el desarrollo de la educación $b$ learning, su alcance y sentido en la configuración de la vida de los docentes, y por extensión de la escuela misma, o mejor de un concepto de escuela, implica considerar la dinámica de una sociedad cambiante, donde las tecnologías se han constituido en medios que ocupan un lugar prominente para los procesos interactivos, configurando entornos importantes que caracterizan a la era del conocimiento y por ende a la comprensión misma del aprendizaje, cómo se hace, cuál es su significado, qué lo constituye.

Este nuevo escenario de integración de métodos y contextos de aprendizaje reconoce los diversos intereses, necesidades y expectativas, no solo de los individuos sino de los diversos grupos con sus características específicas, para definir las sociedades multiculturales.

En esta perspectiva, pensar en la enseñanza y el aprendizaje b-learning en el contexto contemporáneo implica otra forma de ver los espacios y el ámbito educativo. Y de modo especial, implica analizar cómo el contenido del proceso de aprendizaje y de la enseñanza es un elemento representacional de la educación, compartido por los profesores, que interfiere en su práctica profesional.
Una primera unidad de interacción discursiva se configura en torno al lugar y al sentido que tiene en la educación b-learning el concepto y la construcción semántica de las "nuevas tecnologías". Esta construcción pasa por precisar que la calificación de "nuevo" que caracteriza a las tecnologías es un adjetivo de aquellas utilizadas en la época contemporánea y que tienen como soporte particular Internet. Lo que indica que este recurso resulta significativo en el desarrollo de los procesos educativos en contextos b-learning.

Como potencialidades y retos puede destacarse que con el avance de las tecnologías de la información y de la comunicación, la modalidad b-learning estableció un nuevo formato de enseñanza y aprendizaje, también llamado on line, pero asimismo ha venido motivando una crítica y un cuestionamiento a la efectividad y sentido de las dinámicas de los llamados entornos virtuales de aprendizaje, en el que, en la mayoría de casos de este tipo, la educación se ha efectuado (Bairral, 2009, p. 34). Esto deviene como un reto particular justamente porque ha alcanzado un posicionamiento fuerte como modus educativo y mantenerse le obliga a que dicho posicionamiento se dé asimismo en el orden conceptual y epistemológico.

Por otra parte, existe también un cambio en la forma de entender y llevar a cabo la modalidad b-learning, desde un punto de vista legal, dado que se requiere un fuerte rigor en la regulación para satisfacer los criterios de calidad establecidos por la ley, especialmente porque los estudios de grado y postgrado en esta modalidad revela que la tendencia de la educación a distancia en nuestro país se ha convertido en irreversible. $\mathrm{Y}$ en este punto, como señala Mansur (2000), viene a fundarse una fuerte preocupación para demostrar que la distancia constituye una opción con la misma calidad académica de la presencialidad y que se hace necesario romper con la imagen de "segunda categoría" que aún pueda mantenerse sobre ella (p. 67). 
Filatro (2004) establece que entender cómo la información y las tecnologías de la comunicación contribuyen a mejorar los procesos de la enseñanza y de aprendizaje, es una oportunidad para redescubrir la naturaleza particularmente creativa de la educación en el desarrollo humano y social (p. 25). Por su parte, Pires y Veit (2006) destacan que Internet y en general las TIC han representado un hito significativo en la modalidad b-learning a través de la convergencia sincrónica de estas tecnologías y permitiendo nuevas formas de enseñanza y aprendizaje (p. 87).

\section{Las TIC y las representaciones so- ciales del docente.}

Como se indicó atrás, en cuanto al papel que desempeñan los profesores en este escenario, se destaca el diseño de una unidad de comprensión, incluso una red semántica, en parte porque se configuran diversas formas de "alfabetización tecnológica" para movilizar el desarrollo y la construcción del aprendizaje.

Tabla 4.

Representación social del docente

\section{Informante-Actor.}

"En este camino siempre hay "La pedagogía crítica actúa una reacción alérgica, una deliberadamente desde una reacción contraria, pero una perspectiva en la que la envez que conocen como se señanza y el aprendizaje se lleva a cabo esta modalidad, comprometen a expandir, anlo toman con mucha natu- tes que a restringir las oporturalidad incluso con mucho nidades [...]" (Giroux, 2003, entusiasmo" (Participante 5, p. 241).

comunicación personal, 10

de mayo de 2016).

Fuente: elaboración propia.

En este análisis semántico, es posible inferir que en la educación b-learning las tecnologías actuales movilizan la construcción del saber y el desarrollo de los procesos educativos y que dicha movilización pasa por la configuración de una unidad o red de comprensión que involucra disponer a los demás, en la no pre- sencia, para la interacción, las conversaciones, el disenso, el consenso, la argumentación, en últimas, la misma construcción e innovación del saber. Según señalan los participantes, estos aspectos son constituyentes significativos del plan de estudios, de la evaluación, de las estrategias de enseñanza y aprendizaje, en general, de los diversos elementos y componentes que se derivan del horizonte educativo.

La configuración de tal red de comprensión en este escenario de integración de lo "tecnológico" es propicio para la creación de representaciones sociales (Moscovici, 1984, pp. 45-53). En el proceso de análisis de esta investigación, se puede inferir que las tecnologías pueden ser identificadas en las interacciones discursivas entre los tutores y los participantes en los cursos b-learning como un objeto particular de representación social, debido a su carácter movilizador.

Conforme los argumentos desarrollados por Jodelet (2001), las representaciones sociales son construcciones colectivas, fruto de la interacción entre los individuos, integrados en ciertas culturas, que al mismo tiempo llevan a la construcción de una historia social (pp. 17-44). La modificación o cambio en las condiciones de vida de una sociedad hace que dichas representaciones sufran reelaboraciones, transformaciones, cambios en las concepciones sobre los fenómenos que surgen en el horizonte social. El proceso de apropiación de estos fenómenos es un productor de significados e implica lo que Gilly (2001) llama mecanismos de contextualización y descontextualización, que permiten la transmisión social del conocimiento.

En este sentido, los procesos b-learning, como contextos propios en el que devienen representaciones concretas de los docentes, tal es el caso de tutor, sufren continuamente procesos de reelaboración o recontextualización justamente porque la tecnología se ha configurado como un objeto causal y mediador de dichas re- 
presentaciones sociales, y en tanto está provisto de continuas innovaciones, aún más, este hecho es lo que caracteriza su alcance y sentido. Las relaciones en torno a ese objeto constituyen la denominada virtualidad como un punto de inflexión en relación con el perfil contemporáneo del estudiante y el papel del profesor.

La identificación de las representaciones sociales en los procesos b-learning permite configurar el rol y la función de profesores y de los demás participantes en un contexto y área de formación determinados. Dicha configuración del rol profesional está cargada semánticamente de los valores, conceptos, circunstancias subjetivas y objetivas de la vivencia misma que tienen, es decir, los temas, las ideas y los significados contenidos en las representaciones simbólicas revelan los vínculos establecidos por los actores sociales y los recursos utilizados en su ejercicio docente.

\section{Conclusiones}

En el trabajo de investigación realizado se puede concluir que las representaciones sociales de los profesores b-learning tomó como fenómeno fundante la tecnología, en el sentido en que deviene como un generador objeto de las representaciones sociales, movilizando las conversaciones, las opiniones, las actitudes, en general, toda forma de interacción dadas en un grupo.

Los resultados revelaron que la comprensión que tienen los profesores de aquellas formas que se vienen elaborando o constituyendo en torno a las representaciones sociales de sí mismos, están impregnadas de ambigüedad de significado. Y esto es dado porque convergen en el proceso de enseñanza y aprendizaje tanto una dimensión cognitiva, es decir, el saber mismo; una dimensión socio-afectiva, constitución de comunidad; y una dimensión pedagógica, esto es, se pone de manifiesto la pregunta por la planificación, el análisis, la mediación y la reflexión en el proceso de enseñanza.
En este contexto los docentes se han visto expuestos a hacer transformaciones en sus prácticas y se han visto obligados a capacitarse en el uso de estrategias virtuales y en TIC que les permita abrirse a estas nuevas modalidades de enseñanza aprendizaje, sin embargo, se da cierta reticencia frente al tema, pues se les indica que deben diseñar un material útil para entornos de aprendizaje virtuales sin tener previstas unas estrategias didácticas, lo cual termina siendo los mismos materiales de la educación presencial subidos en una plataforma, además existe resistencia al cambio de algunos docentes que no aceptan capacitarse, por lo tanto, se refleja un bajo nivel de formación en competencias en entornos virtuales de aprendizaje y por si fuera poco las universidades no cuentan con unas políticas y disposiciones claras acerca de su uso e implementación.

La modalidad de educación b-learning ha sufrido en los últimos años muchos cambios, tanto en sus objetivos y métodos como en sus instrumentos, redefiniendo el formato de esta modalidad educativa y su ámbito de aplicación.

En este contexto, el progreso continuo y la extensión de la utilización de las tecnologías de la información y las comunicaciones abren nuevas perspectivas para la modalidad b-learning en el sentido, alcance y estructura, por ejemplo, de los entornos virtuales de aprendizaje. Conforme lo enuncian varios participantes, es importante considerar la planificación estructurada y las actividades de desarrollo en la modalidad virtual, especialmente desde una perspectiva de comprensión del saber y del aprendizaje que involucra categorías como la cooperación, la colaboración y la autonomía de los individuos.

En el escenario actual, la modalidad $b$ learning aparece multifacética dada la diversidad de los recursos, perfiles de sujetos y contextos socialmente demandados, lo que plantea la reflexión sobre su oportuna contextualización y actualización. 


\section{Notas}

1 Véase el capítulo I: "La hermenéutica, el camino de la interpretación" (Silva, 2016, pp. 29.

\section{Referencias}

Bairral, M. A. (2009). Tecnologias da Informação e Comunicação na Formação e Educação Matemática (Vol. 1). Rio de Janeiro: Edur.

Barbas, A. (2014). Volver a pensar la educomunicación. En D. Aranda \& A. Creus (XX), Educación, medios digitales y cultura de la participación (pp. 119-135). Barcelona: Universitat Oberta de Catalunya.

Brunner, J. J. (1999). Educación superior en una sociedad global de la información. Bogotá: Universidad Piloto; ASCUN.

Cisterna, F. (2005). Categorización y triangulación como procesos de validación del conocimiento en investigación cualitativa. Theoria, 14(1), 61-71.

Filatro, A. (2004). Design instrucional contextualizado: educação e tecnologia. São Paulo: SENAC.

Gilly, M. (2001). Representaciones sociales en el campo de la educación. En D. Jodelet (Ed.), Representaciones Sociales (pp. 321-341). Río de Janeiro: EDUERJ.

Giroux, H. (1990). Los profesores como intelectuales. Paidós: Barcelona.

Giroux, H. (2001). Cultura, política y práctica educativa. Barcelona: Graó.

Giroux, H. (2003). Pedagogía y política de la esperanza. Madrid: Amorrortu Editores.

Jodelet, D. (Ed.) (2001). Representaciones sociales: un aumento de la superficie. En $R e$ - presentaciones Sociales (pp. 17-44). Río de Janeiro: EDUERJ.

Mansur, A. (2000). La gestión en la educación a distancia: nuevas propuestas, nuevos interrogantes. En E. Litwin (Comp.), La educación a distancia (pp. 53-72). Buenos Aires: Amorrortu Editores.

Moscovici, S. (1979). El Psicoanálisis, su imagen y su público. Buenos Aires: Huemul.

Moscovici, S. (1984). The phenomenon of social representations. En R. Farr \& S. Moscovici (Eds.), Social representations (pp. 18-77). Cambridge: Cambridge University Press.

Oppermann, M. (2000). Triangulation - A Methodological discussion. International Journal of Tourism Research, 2(2), 141-146.

Pires, M., \& Veit, E. (2006). Tecnologias de Informação e Comunicação para ampliar e motivar o aprendizado de Física no Ensino Médio. Revista Brasileira de Ensino Física, 28(2), (pp. 241-248).

Ricoeur, P. (2008). Hermenéutica y acción: de la hermenéutica del texto a la hermenéutica de la acción (Trad. M. Prelooker, $3^{\mathrm{a}}$ ed). Buenos Aires: Prometeo.

Silva, W. (2011). La formación sociocrítica. Una clave para preservar la dignidad de lo humano. Itinerario Educativo, 58, 47-63.

Silva, W. (Ed.) (2016). La hermenéutica, el camino de la interpretación. En Homo capax. Hacia una filosofia de la educación (pp. 29-42). Bogotá:Aula de Humanidades; Universidad de San Buenaventura.

Villanueva, E. (2010). Perspectivas de la educación superior en América Latina: construyendo futuros. Perfiles educativos, 32(129), 86-101. 